\title{
A home-based exercise program for temporomandibular joint osteoarthritis: pain, functionality, and joint structure
}

\author{
Salvador Israel Macías-Hernández ${ }^{1}$, Juan Daniel Morones-Alba ${ }^{2}$, Irene Tapia-Ferrusco ${ }^{3}$, \\ Oscar Benjamín Vélez-Gutiérrez ${ }^{3}$, Cristina Hernández-Diaz ${ }^{4}$, Tania Inés Nava-Bringas ${ }^{5}$, \\ Eva Cruz-Medina ${ }^{1}$, Lya Contreras-del Toro ${ }^{1}$, Ma. de los Angeles Soria-Bastida ${ }^{6}$ \\ ${ }^{1}$ Sports Rehabilitation Departament, Instituto Nacional de Rehabilitación Luis Guillermo Ibarra Ibarra, \\ ${ }^{2}$ Military School of Dentistry, Oral and Maxillofacial Surgery, University of the Army and Air Force of Mexico, \\ ${ }^{3}$ Physical Therapy Division, ${ }^{4}$ Musculoskeletal Ultrasound Laboratory, ${ }^{5}$ Spine Rehabilitation Department, \\ ${ }^{6}$ Orthopedic Rehabilitation Departament, Instituto Nacional de Rehabilitación Luis Guillermo Ibarra Ibarra, Mexico City, Mexico
}

\begin{abstract}
J Korean Assoc Oral Maxillofac Surg 2022;48:50-58)
Objectives: Osteoarthritis (OA) is the most prevalent and disabling joint disease in the world. Temporomandibular joint (TMJ) exercise is a widely used treatment and could be a beneficial and long-term tool for treating TMJ OA. The present study aims to evaluate the effects of therapeutic exercise in the conservative treatment of TMJ OA.

Materials and Methods: A single-group experimental pre-post test was performed. We included patients who met the diagnostic criteria for TMJ OA. Outcome variables were pain intensity (visual analogue scale), functionality (Helkimo index), and structural changes (ultrasound). Follow-up periods were at months 1,3 , and 6 . The intervention included a home-based program with thermotherapy, manual therapy, and therapeutic exercise during the entire follow-up period.

Results: We included 15 patients and 26 joints, all women with a median age of 57 years (range, 49-62 years). Median change in pain intensity on joint palpation, mouth opening, and at rest at the first month was $47.5 \mathrm{~mm}, 51 \mathrm{~mm}$, and $60 \mathrm{~mm}$, respectively, and $48 \mathrm{~mm}, 49.5 \mathrm{~mm}$, and $42.5 \mathrm{~mm}$, at six months $(P=0.001)$. The Helkimo index showed significant improvement in medians from baseline severe dysfunction (17 points) to minimal dysfunction at three and six months ( 2 points) $(P=0.001)$. Ultrasound showed improved disc position.

Conclusion: This study demonstrated significant improvements in pain, function, and joint disc position and represents a valuable tool for the longterm treatment of patients with TMJ OA.
\end{abstract}

Key words: Osteoarthritis, Temporomandibular joint, Excercise, Pain

[paper submitted 2022. 1. 6 / accepted 2022. 1. 26]

\section{Introduction}

Osteoarthritis (OA) is the most prevalent and disabling joint disease globally ${ }^{1}$. In the temporomandibular joint (TMJ), $\mathrm{OA}$ is clinically manifested by pain at rest, upon mouth opening, on direct palpation, and in masticatory muscles, joint

\section{Salvador Israel Macías-Hernández}

Sports Rehabilitation Departament, Instituto Nacional de Rehabilitación Luis Guillermo Ibarra Ibarra, Av. México-Xochimilco 289, Col. Arenal de Guadalupe, Tlalpan, Mexico City 14389, Mexico

TEL: + 52-55-5999-1000

E-mail: drisraelmacias@gmail.com

ORCID: https://orcid.org/0000-0003-1469-6128

(c) This is an open-access article distributed under the terms of the Creative Commons Attribution Non-Commercial License (http://creativecommons.org/ licenses/by-nc/4.0/), which permits unrestricted non-commercial use, distribution, and reproduction in any medium, provided the original work is properly cited.

Copyright (C) 2022 The Korean Association of Oral and Maxillofacial Surgeons. clicking, and limitation of jaw movements. Temporomandibular joint osteoarthritis (TMJ OA) is a frequently undiagnosed subtype of temporomandibular disorder ${ }^{2,3}$.

In TMJ OA, there is radiological evidence of changes in the joint structure, including a reduction or loss of the joint space, flattening or loss of contour of the articular surface, and the appearance of osteophytes, erosions, and sclerosis of the subchondral bone ${ }^{4}$. TMJ OA also affects joint functionality, which in turn affects masticatory functions and can lead to feeding problems and even malnutrition. The Helkimo Index is an internationally validated tool for pathologies that alter TMJ function, particularly temporomandibular dysfunction, that contains information on joint mobility, function, and pain ${ }^{5}$. It is also important to assess joint structure in TMJ OA. Diagnostic ultrasound is a relatively inexpensive, non- 
invasive imaging technique that allows for the accurate assessment of joint dynamics and morphostructural changes in cartilage, articular disc, and subchondral bone. Ultrasound has shown good sensitivity and specificity in detecting internal joint damage compared to magnetic resonance imaging and arthroscopy ${ }^{6}$.

Treatment of TMJ OA is broadly divided into two categories: conservative and invasive. Conservative treatment options include physical therapy, therapeutic exercise, nonsteroidal anti-inflammatory drugs (NSAIDs), and occlusal splints ${ }^{7}$. Invasive treatment includes intra-articular injections, arthrocentesis, arthroscopy, and joint replacement. While these treatments are used in everyday clinical practice, there is currently little high-quality evidence to support their utility, and thus their true efficacy is controversial ${ }^{8}$.

Therapeutic exercise options include exercise and manual therapy. Due to the heterogeneity of these interventions, there is no gold standard or single proposed intervention that is beneficial in this patient population. The need for long-term treatment is a particular concern for OA TMJ patients ${ }^{9}$. From a practical perspective, exercise and manual therapy lend themselves to continued treatment at home because patients can do it themselves without having to visit specialized facilities and because they can be used long-term. These long-term home-based therapies offer many advantages over medication, other physical therapy techniques, or specialized physical agents. The present study aims to evaluate the effects of therapeutic exercise, including thermotherapy and manual therapy in the conservative treatment of OA TMJ.

\section{Materials and Methods}

\section{Design}

An experimental pre-post intervention study was performed including patients of the Instituto Nacional de Rehabilitación Luis Guillermo Ibarra Ibarra in Mexico City, one of Mexico's National Institutes of Health. The study was carried out from January 2017 to February 2020.

\section{Ethics}

The study protocol was registered in the Institutional Research and Ethics Committee of the Instituto Nacional de Rehabilitación derived from the project with the number 49/13. The authors conducted this research in line with the ethical responsibilities established by the World Medical Associa- tion, and all participants provided written informed consent before their inclusion in the study.

\section{Participants}

Patient telephone numbers were obtained from a database provided by the hospital's biostatistics department. All patients diagnosed with TMJ disorder were contacted by telephone, invited to come to the hospital, and examined to confirm that they met the criteria.

\section{Inclusion criteria}

Patients of either sex who were older than 18 years and who met the diagnostic criteria for temporomandibular OA (Research Diagnostic Criteria for Temporomandibular Disorders, Axis I Group IIIb) were eligible. The diagnostic criteria included joint pain at rest or when opening or moving the jaw (identified either by palpation or through patient self-report) and crepitus in the joints with any movement ${ }^{10}$.

In addition, panoramic radiographs, oblique radiographs with the mouth open and closed, and transpharyngeal radiographs were obtained in all patients for radiological classification. The criteria for classification were the reduction of joint space, subchondral sclerosis, flattening of the lateral part of the joint, changes on the surface of the condyle, or presence of osteophytes ${ }^{10}$. One or both joints were included if they met the clinical and radiographic classification criteria: a) crepitus on auscultation of the TMJs, b) chronic joint pain lasting more than six months, c) limited mobility of the mandible with a deviation of the mouth opening to the affected side, d) pain on lateral palpation of the condyle and masseter muscles, e) pain on manual loading of the joint, and f) degenerative changes in the articular bone surfaces and the position of the disc as assessed radiographically.

\section{Exclusion criteria}

Excluded patients were those with a diagnosis of a temporomandibular disorder of any other axis, including other arthropathies, any rheumatic disease affecting the joints (such as rheumatoid arthritis or lupus), with malocclusions, who had used an occlusal splint during the six months before the start of the study, or those who had received intra-articular injections or previous surgical procedures on the jaw or TMJ six months previously.

All clinical evaluations were performed by an oral surgeon 
specializing in TMJ disorders. Radiological evaluations were performed by two independent observers.

\section{Outcomes}

The outcome variables were pain intensity on a visual analogue scale (VAS), functionality with the Helkimo index, and ultrasonographic structural changes.

\section{Procedures}

Patients who were interested in participating after the initial telephone invitation were called in for an examination. The clinical classification criteria were applied, and a full clinical assessment was performed, confirming the clinical criteria; patients also rated themselves on the VAS and the Helkimo index. Those who met the criteria signed an informed consent form. Patients with both painful joints were assessed individually for the right and left joints, i.e., pain and functionality were assessed according to the number of joints included rather than the number of patients.

To confirm that patients met the radiological criteria for $\mathrm{OA}$, radiographs were reviewed by a consultant oral and maxillofacial surgeon and a radiologist expert in musculoskeletal disorders. If both observers independently agreed with the radiological diagnosis, patients were enrolled in the study.

Participants were instructed to discontinue any pharmacological treatment for OA (NSAIDs, glucosamine, chondroitin sulfate, collagen supplements, etc.) seven days before the start of the study and to take acetaminophen as needed and record intake. Taking acetaminophen in doses up to $1 \mathrm{~g}$ every six hours was indicated for moderate to severe pain (greater than $50 \mathrm{~mm}$ at VAS). Patients maintained an intake log in which they recorded acetaminophen intake, dates, and doses.

A fully trained musculoskeletal ultrasound specialist performed baseline ultrasound (with the mouth open and closed). The patient was seated with a neutral head position, with the probe at the level of the zygomatic arch and the TMJ. The structure was observed as a homogeneous linear hyper- or hypoechoic image, the normal being a homogeneous linear image and the abnormal being a heterogeneous nonlinear image. The disc was observed with the mouth closed and then marked as observed or not observed (the disc is observed as a thin hypoechoic line leaving a halo over the condyle). Measurements of the mouth opening joint were taken from the mouth closed to the mouth open from bone edge to bone edge. Power Doppler was recorded as either having a signal present or absent; the presence of osteophytes, enthesophytes, or other visible lesions was also recorded.

Follow-up was done at months one, three, and six with clinical assessment, administration of the same VAS and the Helkimo index, and ultrasound.

\section{Intervention}

The patients were trained in one session to learn the entire program and were instructed to continue the exercises 3-5 times a week, once a day, during the six-month follow-up. Two previously trained physical therapists were responsible for teaching patients, and the following program was used:

\section{1) Patient in a supine position}

Application of superficial heat with a heat pack covering the jaw area for 15 minutes.(Fig. 1. D)
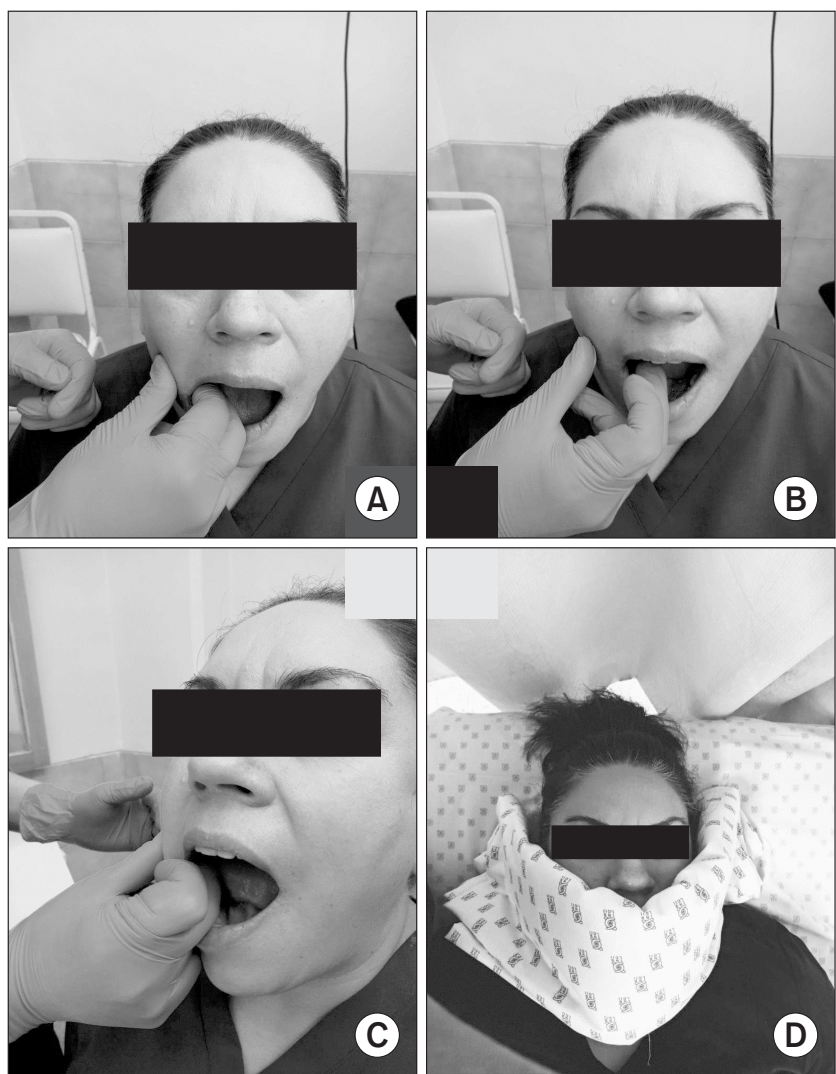

Fig. 1. A. Intraoral massage with circular movements of index and middle fingers in a clockwise direction. B. Intraoral massage with circular movements of the middle finger in a counterclockwise direction. C. Kneading the inner muscles of the cheeks, stroking from the base to the apex for protrusion and retraction. D. Hot pack placing prior the exercise performance.

Salvador Israel Macías-Hernández et al: A home-based exercise program for temporomandibular joint osteoarthritis: pain, functionality, and joint structure. J Korean Assoc Oral Maxillofac Surg 2022 

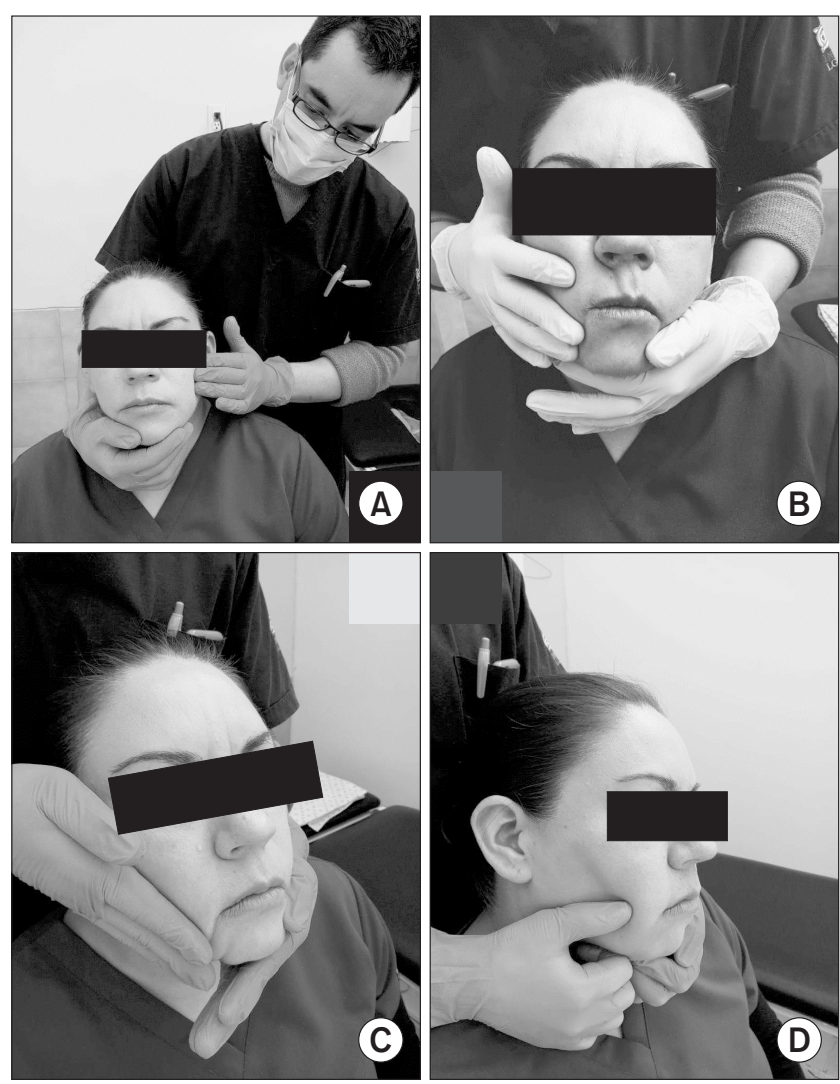

Fig. 2. External massage. A. Up and down movements. B. Circling and kneading movements. C. Gliding and sliding massage movements with full hand. D. Thumb gliding movements.

Salvador Israel Macías-Hernández et al: A home-based exercise program for temporomandibular joint osteoarthritis: pain, functionality, and joint structure. J Korean Assoc Oral Maxillofac Surg 2022

2) Patient in a seated position

(1) Intraoral massage was performed with the thumb outside the mouth and the index or middle finger (both optional) inside the mouth. Ten repetitions were made, starting with: Circular movements to the inner muscles of the cheeks, kneading the inner muscles of the cheeks and stroking from the base to the apex for protrusion and retraction, shown by the therapist but performed by the patient itself at home.(Fig. 1. A-C)

(2) External massage: A lubricant (oil) was used to cover the jaw area and the entire lateral area of the face. Ten repetitions of gliding, circling, and kneading movements were performed and concluded with a neck massage.(Fig. 2)

(3) Stretching: The patient was asked to open their mouth. With the mouth open, the cheek was grasped from the inside with the index, middle, or both fingers, and a slight deviation was made outward. The patient was asked to close their mouth partially. They maintained the outward deviation for 10 seconds, rested for 5 seconds, and repeated the stretch
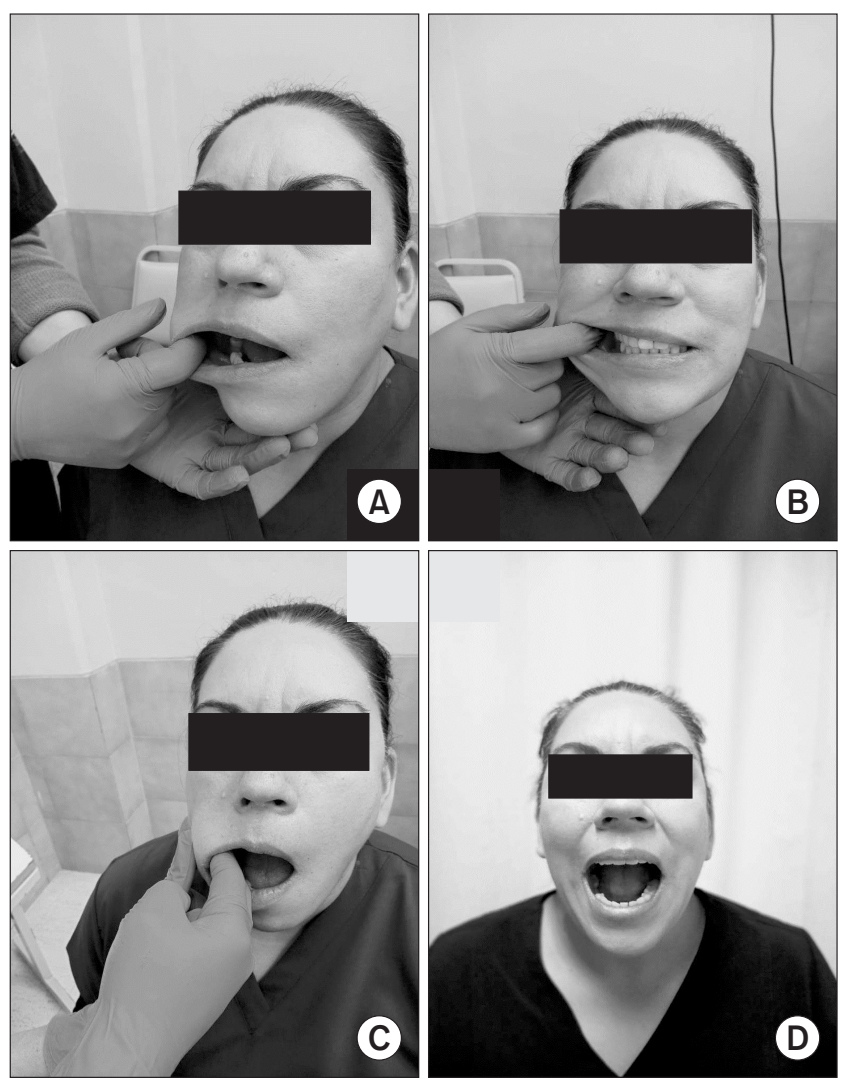

Fig. 3. A, B. Patient with the mouth open grasped from the inside with the index finger in two positions. C. Grasp from the inside with two fingers and slight deviation. D. Sustained maximum tolerated mouth opened.

Salvador Israel Macías-Hernández et al: A home-based exercise program for temporomandibular joint osteoarthritis: pain, functionality, and joint structure. J Korean Assoc Oral Maxillofac Surg 2022

This was done three times on each side of both cheeks. The patient opened their mouth as wide and gently as possible without causing pain. The opening was held for 10 seconds, then rested for 5 seconds, and the procedure was repeated three times, again shown by the therapist but performed by the patient itself at home.(Fig. 3)

(4) Free joint mobilization: Ten repetitions of each movement without causing pain, including descensus and elevation (opening and closing the mouth), protrusion and retrusion (forward and backward projection), and laterality (right, left). (Fig. 4)

(5) Strengthening exercises: The patients performed three repetitions and held each repetition for 10 seconds with a 5 -second rest. These exercises included descent, placing the hand on the lower part of the chin, and trying to open the mouth without letting it close; pressure was maintained for 10 seconds.(Fig. 5. A) Patients advanced to placing their hand in front of their chin and pushing their chin forward for 10 

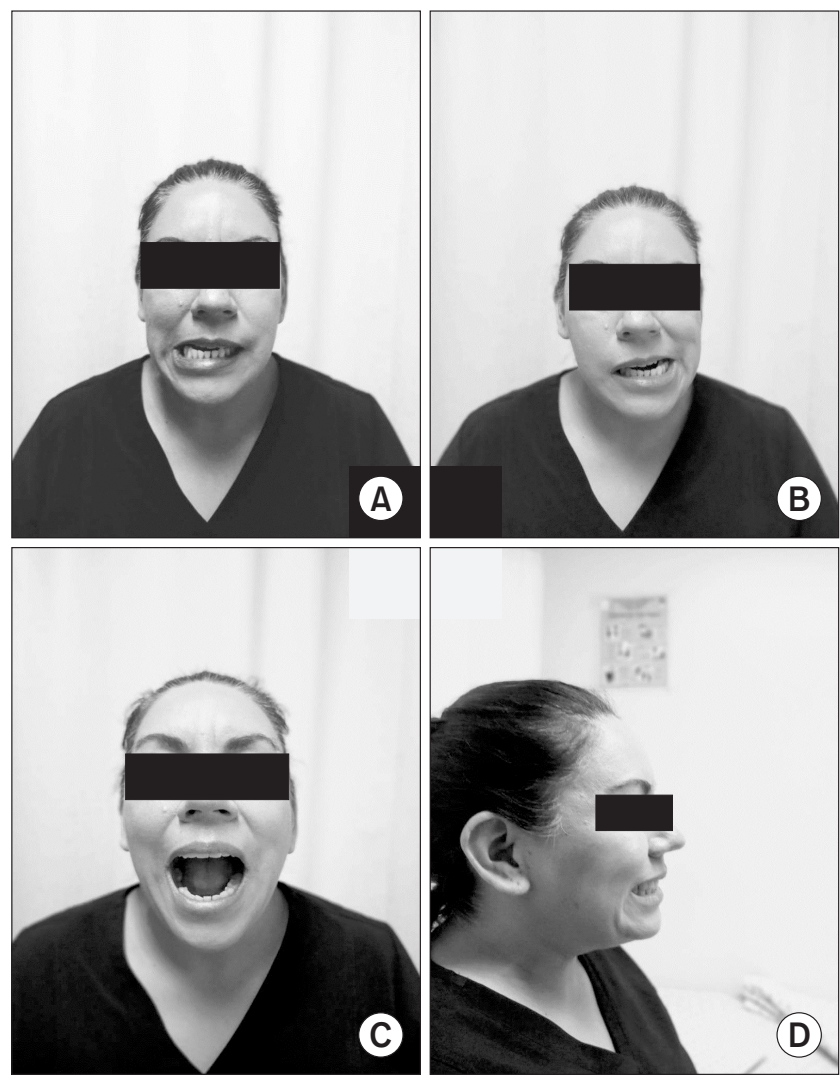

Fig. 4. A, B. Lateral right and left mobilization. C. Descensus and elevation (opening and closing the mouth). D. Protrusion and retrusion (forward and backward projection).

Salvador Israel Macías-Hernández et al: A home-based exercise program for temporomandibular joint osteoarthritis: pain, functionality, and joint structure. J Korean Assoc Oral Maxillofac Surg 2022

seconds (being careful not to do this with the neck muscles). (Fig. 5. B) In lateralization, the patient places their hand on one side of their chin and makes a lateral movement in the direction of the hand that offers resistance.(Fig. 5. C) For elevation, the patient places a pencil between the teeth (molars) and presses for 10 seconds.(Fig. 5. D)

In an endeavor to improve exercise adherence, all participants kept an exercise diary where they recorded the number of exercises performed, type, and their signature verifying completion. Patients were also called weekly to ask questions about the exercises and to encourage their performance. They were given an exercise program with outlines and a written description to take home.

\section{Statistical analysis}

Descriptive statistics were obtained and summarized as median and interquartile range. A chi-square or Fisher's exact test was performed to analyze the difference between groups
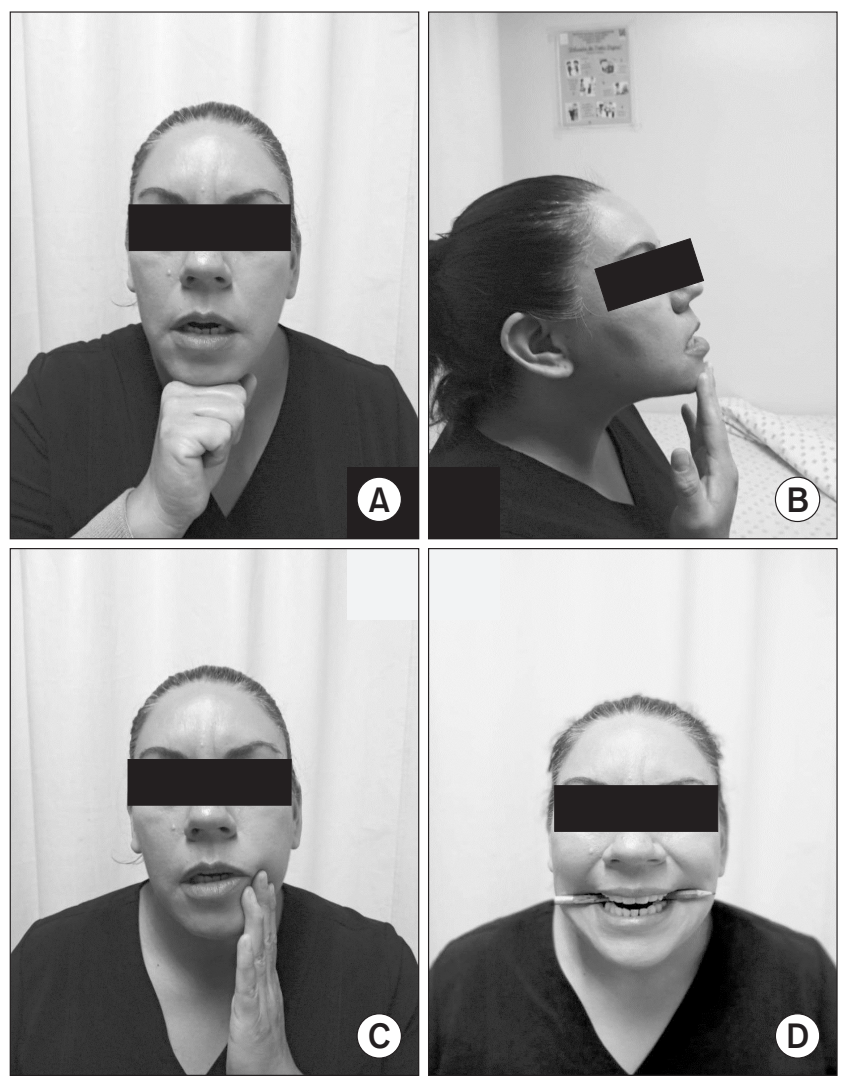

Fig. 5. A. Descent placing the hand on the lower part of the chin and trying to open the mouth without letting it close. B. Patient advanced to placing her hand in front of their chin and pushing their chin forward. C. The patient places her hand on one side of her chin and makes a lateral movement in the direction of the hand that offers resistance. D. For elevation, the patient places a pencil between the teeth (molars) and presses.

Salvador Israel Macías-Hernández et al: A home-based exercise program for temporomandibular joint osteoarthritis: pain, functionality, and joint structure. J Korean Assoc Oral Maxillofac Surg 2022

in terms of the qualitative variables. To look for differences in the ranges of the quantitative variables, a Mann-Whitney $\mathrm{U}$ test was performed for independent variables, the Wilcoxon test for pairs of related variables, and the changes over time in the four measurements were analyzed using the Friedman test within each group. The alpha significance level taken was 0.05. The IBM SPSS Statistics software (ver. 19.0; IBM, Armonk, NY, USA) was used.

\section{Results}

Fifty-two patients with symptoms of chronic pain in one or both TMJs were recruited, invited to the hospital, and had radiologic projections obtained. Thirty-seven patients were excluded because they did not meet radiographic criteria. Fifteen patients and 26 joints that met both clinical and ra- 
diological criteria were included in the analysis. All patients were women. The median age was 57 years (range, 49-62 years). There were no losses to follow-up, and all patients completed their treatment. A frequency of more than three times per week was observed in all cases, with an average of $4.52 \pm 1.23$ times per week at the three-month follow-up and $3.62 \pm 0.41$ times per week at the six-month follow-up.

Table 1 shows pain levels; a notable decrease in pain is apparent after the first month of treatment. This decrease was maintained at the six-month follow-up, with no differences detectable by a Student $t$-test between 1-3 or 3-6 months, but with a difference between 1-6 months. Pain intensity was measured at joint palpation, mouth opening, and rest. Pain levels went from a median of $47.5 \mathrm{~mm}, 51 \mathrm{~mm}$, and $60 \mathrm{~mm}$, respectively, at the first-month assessment to $48 \mathrm{~mm}, 49.5$ $\mathrm{mm}$, and $42.5 \mathrm{~mm}$, respectively, at the six-month assessment.

Functionality was measured by the Helkimo index using the sub-indexes for mobility, TMJ function, muscle pain, TMJ pain, and pain on mandibular movement, each of which was assigned a score from 0 to 5 . The total score is interpreted as follows: 0 , free of TMJ problems; $1-4$ points, minimal dysfunction; 15-17 points, severe dysfunction. The results are summarized in Table 2. A significant improvement in joint functionality was observed in the first month of treatment and maintained after 3 and 6 months. For the baseline total score results, the medians were severe dysfunction (17 points), free of TMJ problems at the first month ( 0.25 points), and minimal dysfunction at 3 and 6 months ( 2 points).

Table 1. Visual analogue scale results for different follow-up periods (unit: $\mathrm{mm}$ )

\begin{tabular}{|c|c|c|c|c|c|c|c|c|c|}
\hline & Baseline & $1-\mathrm{mo} \mathrm{F} / \mathrm{U}$ & $P$-value & 3-mo F/U & $\begin{array}{l}P \text {-value } \\
(1-3 \text { mo })\end{array}$ & 6-mo F/U & $\begin{array}{l}P \text {-value } \\
(3-6 \text { mo })\end{array}$ & $\begin{array}{c}\text { Median change } \\
1-6 \text { mo }\end{array}$ & $\begin{array}{l}P \text {-value } \\
(1-6 \text { mo })\end{array}$ \\
\hline Palpation & $72(55-92)$ & $24.5(0-39)$ & $<0.001$ & $32(0-34.5)$ & 0.12 & $24(0-38)$ & 0.43 & $48(40-82)$ & 0.001 \\
\hline Opening & $69.5(61-83.25)$ & $18.5(0-37)$ & 0.001 & $29(0-50.25)$ & 0.27 & $20(0-51)$ & 0.48 & $49.5(42.5-70)$ & 0.001 \\
\hline $\begin{array}{l}\text { Mouth closed } \\
\text { resting }\end{array}$ & $62.5(55-84)$ & $5(0-42)$ & 0.001 & $25(0-42.5)$ & 0.17 & $20(0.42)$ & 0.52 & $42.5(40-78)$ & 0.001 \\
\hline
\end{tabular}

(F/U: follow-up)

Values are presented as mean (range) or median (range).

Salvador Israel Macías-Hernández et al: A home-based exercise program for temporomandibular joint osteoarthritis: pain, functionality, and joint structure. J Korean Assoc Oral Maxillofac Surg 2022

Table 2. Helkimo index results on functionality

\begin{tabular}{|c|c|c|c|c|c|c|c|c|}
\hline & Baseline & $1 \mathrm{mo}$ & $P$-value & $3 \mathrm{mo}$ & $\begin{array}{l}P \text {-value } \\
(1-3 \text { mo })\end{array}$ & $6 \mathrm{mo}$ & $\begin{array}{l}P \text {-value } \\
(3-6 \text { mo) }\end{array}$ & $\begin{array}{c}P \text {-value } \\
(1-6 \text { mo })\end{array}$ \\
\hline Mobility index & $3(0-5)$ & $0.25(0-1)$ & 0.001 & $0.5(0-5)$ & 0.87 & $1(0-5)$ & 0.72 & 0.01 \\
\hline TMJ function & $3.8(0-5)$ & $0.5(0-1)$ & 0.001 & $1(0-5)$ & 0.71 & $0.5(0-5)$ & 0.65 & 0.001 \\
\hline Muscle pain & $3.2(1-5)$ & $0.5(0-5)$ & 0.005 & $0.5(0-1)$ & 0.43 & $0.5(0-5)$ & 0.52 & 0.005 \\
\hline TMJ pain & $3.4(1-5)$ & $0(0-5)$ & 0.001 & $0(0-1)$ & 0.32 & $0(0-5)$ & 0.82 & 0.001 \\
\hline $\begin{array}{l}\text { Pain on mandible } \\
\text { movement }\end{array}$ & $3.6(1-5)$ & $0(0-5)$ & 0.001 & $0(0-1)$ & 0.23 & $0(0-1)$ & 0.72 & 0.001 \\
\hline Total score & $17(13-17)$ & $0.25(2-7)$ & $<0.0001$ & $2(0-3.75)$ & 0.44 & $2(0-8)$ & 0.59 & 0.001 \\
\hline
\end{tabular}

(TMJ: temporomandibular joint)

Values are presented as mean (range).

The total score is interpreted as follows: 0, free of TMJ problems; 1-4 points, minimal dysfunction; 15-17 points, severe dysfunction.

Salvador Israel Macías-Hernández et al: A home-based exercise program for temporomandibular joint osteoarthritis: pain, functionality, and joint structure. J Korean Assoc Oral Maxillofac Surg 2022

Table 3. Ultrasound measurements

\begin{tabular}{|c|c|c|c|c|c|}
\hline & Baseline & $3 \mathrm{mo}$ & $P$-value & $6 \mathrm{mo}$ & $P$-value \\
\hline Closed mouth (mm) & $5.1(5.3-7.4)$ & $5.2(4.0-6.1)$ & 0.43 & $5.3(5.2-6.5)$ & 0.83 \\
\hline Open mouth (mm) & $8.1(6.8-13.2)$ & $8.5(7.1-9.5)$ & 0.05 & $9.1(8.4-13.5)$ & $0.04^{1}$ \\
\hline Difference $(\mathrm{mm})$ & $3.0(1.5-5.8)$ & $3.3(3.1-3.4)$ & 0.12 & $3.8(3.2-7)$ & $0.04^{1}$ \\
\hline Joint disc & & & 0.06 & & 0.02 \\
\hline Non-visible & $16(61.5)$ & $14(53.8)$ & & $11(42.3)$ & \\
\hline Visible & $10(38.5)$ & $12(46.2)$ & & $15(57.7)$ & \\
\hline
\end{tabular}

${ }^{1}$ Baseline: 6 months.

Values are presented as mean (range) or number (\%).

Salvador Israel Macías-Hernández et al: A home-based exercise program for temporomandibular joint osteoarthritis: pain, functionality, and joint structure. J Korean Assoc Oral Maxillofac Surg 2022 
Structural changes were measured by ultrasound; ultrasounds were examined for echogenicity of structure in general, visibility of articular discs, Doppler, presence of synovitis and osteophytes, and distances measured with the mouth closed and open. All joints were linearly homogeneous from baseline and at follow-up, as were the presence of Doppler, which was absent in all cases, and the presence of synovitis. The presence of osteophytes was detected in two joints (7.7\%) using this method, and these did not change on follow-up. The changes in the observation of the disc joint are shown in Table 3, where a difference in the proportion of observation of the disc joint by the ultrasound method is shown. Table 3 also shows changes in the measurements of the opening distances with a difference between baseline and at 3 and 6 months.

As for the radiological findings, reduction of the joint space was most common in 24 joints $(92.3 \%)$, presence of osteophytes in 16 joints (61.5\%), subchondral sclerosis in 14 joints $(53.8 \%)$, flattening of the lateral part of the joint in 10 joints $(38.5 \%)$, and changes on the surface of the condyle in 7 joints (26.9\%).

In terms of NSAIDs, in the first month, patients reported the use of paracetamol for pain in any joint at the prescribed dose ( $1 \mathrm{~g}$ every six hours), and three patients (20.0\%) reported use due to pain in five joints (19.2\%). At the three-month evaluation, no patient reported the use of acetaminophen, and in the 6-month evaluation, four patients $(26.7 \%)$ reported analgesic use in six joints (23.1\%). The average use was short, only five doses (range, 2-10 doses), with a median (interquartile range) of 1.25 days (range, $0.5-2.5$ days), with no significant changes in medication use during follow-up $(P=0.12)$ in months 1-6.

\section{Discussion}

This study demonstrates the usefulness of a home-based therapeutic exercise program as part of the treatment for TMJ OA. This intervention was applied to patients with a specific diagnosis of TMJ OA that included clinical and radiological criteria, not just TMJ disorders, and included a functional and structural evaluation. To our knowledge, few studies include the results of this type of treatment in patients with a specific clinical and radiological diagnosis of TMJ OA and with outcomes that combine pain, functionality, and joint structure.

The use of NSAIDs is the first choice in the treatment of TMJ OA. However, their long-term use is not recommended due to the potentially harmful effects, as TMJ OA is a chronic disease with morphological changes of the joint. Therapeutic exercise is a more useful tool in the long term ${ }^{11}$.

Our intervention included a complete home-based program including superficial heat, massage therapy, joint mobilization, stretching, and strengthening. This proposed program can be completely and easily performed at home in the long term, which is useful in a condition such as symptomatic OA where structural changes in the joint are known to occur. Thus, it could be a good alternative for patients before invasive interventions are required. Our results show that patients tolerate the program well and maintain it without discontinuing it during the follow-up period. This reinforces our belief that this is an ideal program that can be implemented in the medium and long term.

Our study shows quantification of pain at rest, upon joint palpation, and at maximum mouth opening. The most significant change in pain intensity occurred after the physiotherapy intervention in the first month, in which there was a decrease of $57.5 \mathrm{~mm}$ in resting pain and $47.5 \mathrm{~mm}$ in maximum mouth opening, corresponding to a decrease between $67.5 \%$ and $92 \%$ of the baseline pain intensity, and then remained mild through the end of the six-month follow-up period.

In a controlled clinical trial published by Tuncer et al. ${ }^{12}$, when comparing home physical therapy alone or additional manual therapy in a facility, both groups saw significant improvement in pain. However, pain on exertion (palpation) decreased significantly in favor of the manual therapy group. In contrast to our study, they reported patients with TMJ symptoms of different etiologies with a one-month follow-up. Our study included a complete home-based therapy program with follow-up at six months. We also described the intervention in detail so that it is available and can be replicated by clinicians or researchers ${ }^{12}$.

A review paper published by Shimada et al. ${ }^{13}$ summarized the results of various exercise modalities for TMJ disorders. They found that - in general - passive and active mobilization exercises and postural exercises reduced joint and muscle pain. Of course, methodological flaws in study design and treatment standardization makes comparing studies difficult, in addition to the fact that conditions are not classified and are not specifically diagnosed. The present study also included only patients with OA classification and confirms what is described in this review, that exercise is not only effective in reducing pain, but also improving function and joint structure ${ }^{13}$.

Another systematic review with a meta-analysis on the effectiveness of manual therapy and exercise for TMJ disorders included 48 randomized clinical trials. This review reported 
a large degree of heterogeneity in diagnoses, including different disease classification criteria and types of intervention. While results are mixed, most did mention a benefit to the use of exercise. The review includes a meta-analysis of manual therapy plus exercise, and the five studies that were included showed a significantly better effect size compared to other interventions (such as medication alone and the use of splints) at a follow-up of one to three months. This effect is confirmed in our current study and observed in a selected group of patients with $\mathrm{OA}^{14}$.

Another systematic review published by Cochrane Library on interventions for the treatment of TMJ OA only included interventions such as hyaluronic acid, steroid injections, NSAIDs, and occlusal splints. Exercise and physical therapy were not included due to the poor quality of evidence in the published studies. Although our study is not a controlled clinical trial, it has a consistent and well-classified group of individuals, which makes the information valuable ${ }^{15}$. Also, our study shows that the need to take NSAIDs was low and only for short periods.

The Helkimo index assesses several functional aspects of the joint, specifically mobility, mouth opening, and joint and muscle pain. Significant improvements were observed in all areas, especially in the first month of treatment, from severe dysfunction to minimal dysfunction, which was maintained throughout the follow-up period. This means that patients showed a significant improvement in functionality in all measured points.

In a study with a design similar to ours involving 12 subjects with mixed TMJ disorders, Calixtre et al. ${ }^{16}$ tested an exercise program that included manual therapy, mobilization, and stretching. After a 5-week follow-up period, they assessed pain and functionality with the program and found a significant increase in functionality and a decrease in pain, although unlike ours, their study had a smaller sample, shorter follow-up period, and mixed TMJ disorders ${ }^{16}$.

Ultrasound imaging did not show any siphoning changes in the echogenicity of the tissues or in the linearity of the image, which shows that this type of therapy improves the pain and the functional state of the joint, although it most likely has no structural effect on the cartilage joint. It is noticeable that the visualization of the disc changed significantly after six months, as it was observed in about $38.5 \%$ of cases at baseline and, after six months, in $57.7 \%$ of cases. This could be because the position of the disc joint changed due to the intervention, and it may have been rearranged after the subluxation. This finding is important because, in addition to the musculoskeletal effects on pain, inflammation, and mobility, there are also effects related to the position of the disc joint ${ }^{17}$.

Another significant result was the change in mouth opening on ultrasound, which was significant at six months. Patients were able to achieve a larger mouth opening after intervention, which was likely associated with decreased pain and muscle contracture.

\section{Conclusion}

The home-based exercise program we proposed led to improvement in pain at rest, on palpation, and improvement of pain in mouth opening. In addition, functional improvement of the joint was noted. Therefore, this program represents a cost-effective treatment with minimal side effects accessible to all patients with TMJ OA.

The lack of a control group meant that this study was not a clinical trial. However, a very large effect size was still observed with the intervention. Another weakness was that this study had a relatively small sample size, due in part to the inclusion of only patients who met both clinical and radiographic criteria. In the future, a controlled clinical trial with a larger sample size should be conducted to verify the effect and improve the quality of the evidence.

\section{ORCID}

Salvador Israel Macías-Hernández, https://orcid.org/00000003-1469-6128

Juan Daniel Morones-Alba, https://orcid.org/0000-00023961-5243

Irene Tapia-Ferrusco, https://orcid.org/0000-0001-9891-2005

Oscar Benjamín Vélez-Gutiérrez, https://orcid.org/00000002-1700-9488

Cristina Hernández-Diaz, https://orcid.org/0000-00019020-3722

Tania Inés Nava-Bringas, https://orcid.org/0000-00032601-0621

Eva Cruz-Medina, https://orcid.org/0000-0001-9867-6102

Lya Contreras-del Toro, https://orcid.org/0000-0001-5004-5176

Ma. de los Angeles Soria-Bastida, https://orcid.org/00000002-7842-4247

\section{Authors' Contributions}

All authors actively participated in the study and made substantial contributions to this article. S.I.M.H. participated 
in conception, design, data acquisition, analysis and data interpretation, drafting, and final approval. J.D.M.A. participated in design, data acquisition, data interpretation, analysis (radiographic), drafting, critical revision, and final approval. I.T.F. and O.B.V.G. participated in data acquisition, intervention (physical therapy), data interpretation, drafting, critical revision, and final approval. C.H.D. participated in data acquisition, data interpretation (ultrasound), drafting, critical revision, and final approval. T.I.N.B., E.C.M., L.C.T., and M.A.S.B. participated in data acquisition, data interpretation, drafting, critical revision, and final approval.

\section{Ethics Approval and Consent to Participate}

The study protocol was registered in the Institutional Research and Ethics Committee of the Instituto Nacional de Rehabilitación derived from the project with the number 49/13. All participants provided written informed consent before their inclusion in the study.

\section{Consent for Publishing Photographs}

Written informed consent was obtained from the patients for publication of this article and accompanying images.

\section{Conflict of Interest}

No potential conflict of interest relevant to this article was reported.

\section{References}

1. Sharma L. Osteoarthritis of the knee. N Engl J Med 2021;384:519. https://doi.org/10.1056/NEJMcp1903768

2. Kalladka M, Quek S, Heir G, Eliav E, Mupparapu M, Viswanath A. Temporomandibular joint osteoarthritis: diagnosis and long-term conservative management: a topic review. J Indian Prosthodont Soc 2014;14:6-15. https://doi.org/10.1007/s13191-013-0321-3

3. Liu Y, Wu J, Fei W, Cen X, Xiong Y, Wang S, et al. Is there a difference in intra-articular injections of corticosteroids, hyaluronate, or placebo for temporomandibular osteoarthritis? J Oral Maxillofac Surg 2018;76:504-14. https://doi.org/10.1016/j.joms.2017.10.028

4. Whyte A, Boeddinghaus R, Bartley A, Vijeyaendra R. Imaging of the temporomandibular joint. Clin Radiol 2021;76:76.e21-35. https://doi.org/10.1016/j.crad.2020.06.020

5. Rani S, Pawah S, Gola S, Bakshi M. Analysis of Helkimo index for temporomandibular disorder diagnosis in the dental students of
Faridabad city: a cross-sectional study. J Indian Prosthodont Soc 2017;17:48-52. https://doi.org/10.4103/0972-4052.194941

6. Iagnocco A, Naredo E. Ultrasound of the osteoarthritic joint. Clin Exp Rheumatol 2017;35:527-34.

7. Al-Ani Z. Temporomandibular joint osteoarthrosis: a review of clinical aspects and management. Prim Dent J 2021;10:132-40. https://doi.org/10.1177/2050168420980977

8. Derwich M, Mitus-Kenig M, Pawlowska E. Interdisciplinary approach to the temporomandibular joint osteoarthritis-review of the literature. Medicina (Kaunas) 2020;56:225. https://doi.org/10.3390/ medicina56050225

9. Wright EF, Klasser GD. Manual of temporomandibular disorders. 4th ed. Hoboken (NJ): Wiley-Blackwell; 2019.

10. Schiffman E, Ohrbach R, Truelove E, Look J, Anderson G, Goulet JP, et al.; International RDC/TMD Consortium Network, International Association for Dental Research; Orofacial Pain Special Interest Group, International Association for the Study of Pain. Diagnostic criteria for temporomandibular disorders (DC/TMD) for clinical and research applications: recommendations of the International RDC/TMD Consortium Network* and Orofacial Pain Special Interest Group ${ }^{\dagger}$. J Oral Facial Pain Headache 2014;28:6-27. https://doi.org/10.11607/jop.1151

11. Mejersjö C, Wenneberg B. Diclofenac sodium and occlusal splint therapy in TMJ osteoarthritis: a randomized controlled trial. J Oral Rehabil 2008;35:729-38. https://doi.org/10.1111/j.13652842.2008.01863.x

12. Tuncer AB, Ergun N, Tuncer AH, Karahan S. Effectiveness of manual therapy and home physical therapy in patients with temporomandibular disorders: a randomized controlled trial. J Bodyw Mov Ther 2013;17:302-8. https://doi.org/10.1016/j.jbmt.2012.10.006

13. Shimada A, Ishigaki S, Matsuka Y, Komiyama O, Torisu T, Oono $\mathrm{Y}$, et al. Effects of exercise therapy on painful temporomandibular disorders. J Oral Rehabil 2019;46:475-81. https://doi.org/10.1111/ joor. 12770

14. McNeely ML, Armijo Olivo S, Magee DJ. A systematic review of the effectiveness of physical therapy interventions for temporomandibular disorders. Phys Ther 2006;86:710-25.

15. de Souza RF, Lovato da Silva CH, Nasser M, Fedorowicz Z, Al-Muharraqi MA. Interventions for the management of temporomandibular joint osteoarthritis. Cochrane Database Syst Rev 2012;2012:CD007261. https://doi.org/10.1002/14651858. CD007261.pub2

16. Calixtre LB, Grüninger BL, Haik MN, Alburquerque-Sendín F, Oliveira AB. Effects of cervical mobilization and exercise on pain, movement and function in subjects with temporomandibular disorders: a single group pre-post test. J Appl Oral Sci 2016;24:188-97. https://doi.org/10.1590/1678-775720150240

17. Razek AA, Al Mahdy Al Belasy F, Ahmed WM, Haggag MA. Assessment of articular disc displacement of temporomandibular joint with ultrasound. J Ultrasound 2014;18:159-63. https://doi. org/10.1007/s40477-014-0133-2

How to cite this article: Macías-Hernández SI, Morones-Alba JD, Tapia-Ferrusco I, Vélez-Gutiérrez OB, Hernández-Diaz C, NavaBringas TI, et al. A home-based exercise program for temporomandibular joint osteoarthritis: pain, functionality, and joint structure. J Korean Assoc Oral Maxillofac Surg 2022;48:50-58. https://doi. org/10.5125/jkaoms.2022.48.1.50 